\title{
Performance Evaluation of Bit Loading, Subcarrier Allocation, and Power Control Algorithms for Cellular OFDMA Systems
}

\author{
Slawomir Pietrzyk ${ }^{1}$ and Gerard J.M. Janssen ${ }^{2}$ \\ 1 Access Network Unit, Polska Telefonia Cyfrowa Sp. z o.o., Warsaw, Poland, \\ S.Pietrzyk@ieee.org \\ 2 Wireless \& Mobile Communications Group, Dept. of Electr. Eng., Math. and \\ Comp. Sc. (EEMCS), Delft University of Technology, Delft, The Netherlands, \\ G.Janssen@ewi.tudelft.nl
}

\begin{abstract}
We consider the problem of radio resource allocation for QoS support in the downlink of a cellular OFDMA system. The major impairments present are co-channel interference (CCI) and frequency selective fading. The allocation problem involves assignment of base stations, subcarriers and bits, as well as power control, for multiple users. We evaluate the performance of a three-stage, low-complexity, heuristic algorithm, which allows to distribute radio resources among multiple users according to theirs individual QoS requirements, while at the same time maintaining the QoS of already established links in all co-channel cells. The evaluation includes checking system operation for various conditions described by different: a) delay spread, b) data rate required by a single user and c) path loss. It is shown that the proposed method is superior in terms of offered traffic and blocking probability to classical method based on FDMA with power control. Also, the performance of our scheme increases in highly frequency selective environment, disastrous for classical fixed schemes, since our method benefits from multiuser diversity.
\end{abstract}

\section{Introduction}

The cellular environment poses certain challenges to the resource allocation process, namely the needs: a) to handle co-channel interference (CCI) caused by the $\mathrm{RF}$ bandwidth reuse, b) to provide and maintain individual QoS profiles required by multiple users, and finally c) to assign radio resources efficiently. In case of cellular OFDMA system, resource allocation includes, besides OFDM subcarrier assignment, also assignment of modulation orders (bit loading), power levels and base stations (access points) serving the users, 1]. In order to meet these challenges, we apply a dynamic resource allocation approach, which requires the knowledge of channel conditions. In [2], it is claimed that such a design methodology results in higher system performance when compared to interference-averaging techniques (such as CDMA) and much higher performance when compared to fixed resource allocation methods (such as e.g. fixed frequency planning). 
Previous work in the area of resource allocation for OFDMA systems that constitutes the base for this paper can be classified into the following groups of papers.

- Single-cell systems, [3], 4], 5]. These papers present various interesting resource allocation algorithms for OFDMA but they do not consider CCI.

- Cellular systems, 6], 7], [8]. Here, methods for OFDMA resource allocation in cellular environment are proposed. However, the considered algorithms do not allow for maintenance of QoS in the co-channel cells. CCI is limited rather by interference avoidance than control.

- Non-cellular systems with CCI control, 9], [10]. In these papers a mature consideration of a problem of bit, subcarrier and power allocation for OFDMA under CCI is given. However, the presented solutions exhibit quite high complexity and are suitable for point-to-point networks, such as xDSL or fixed wireless access, where allocation of transmitters to receivers is predetermined.

Our previous work in the area of resource allocation for OFDMA in cellular environment includes [11] and [12]. In [12] we proposed algorithms for appointing a serving base station, allocation of subcarriers, adaptation of modulation levels (bit loading), and finally control of transmit power to satisfy and maintain the users' individual QoS requirements, expressed in terms of bit rate and bit error rate, at the lowest possible cost of resource utilization in OFDMA system. In this paper, in conjunction with the ideas from the companion paper [12] we have focused on performance evaluation of the proposed algorithms under various settings of system parameters. This analysis allows us to better understand applicability of the proposed methods. Moreover, in this paper we propose a modification of the algorithms described in [12] to allow for performing adaptive cell selection (ACS) and we evaluate its performance by simulations. ACS was considered in an OFDMA scenario in [7] but its forms are known in contemporary systems (e.g. cell selection/reselection in GSM). Thanks to ACS, if a candidate cell is unable to serve a user, another cell is tried from the list of preferred cells. In this way, we increase the chances for user admission.

The paper is organized as follows. Section 2 presents the considered system model and formulates the allocation problem. Section 3 presents the proposed solution, while Section 4 verifies its performance by numerical experiments. Finally, conclusions are given in section 5 .

\section{System Model and Problem Formulation}

The considered downlink of a cellular OFDMA system consists of $K$ cells, each with one base station (BS) serving in total (i.e. in the entire system) $U$ users, as shown in Figure 1 and in Figure 2. The total available bandwidth $B W$ is partitioned into $N$ narrowband OFDM subcarriers. In principle, the entire bandwidth $B W$ is available in every cell (i.e. a reuse of one is applied) and the selection of a particular subcarrier is subject to local load and channel conditions. This 


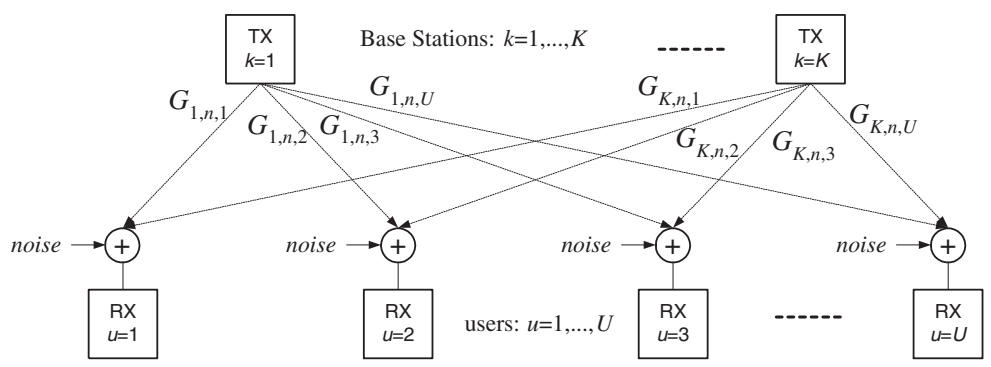

Fig. 1. Model of a downlink cellular OFDMA system for subcarrier $n$, where $n=$ $1, . ., N$. Dotted lines indicate possible options for connecting transmitters (TX) with receivers (RX)

methodology is especially applicable to systems with a non-uniform spatial distribution of traffic and it does not require frequency planning, which is usually a complex task for the network operator. The bandwidth of each subcarrier is chosen to be sufficiently smaller than the coherence bandwidth of the channel in order to prevent inter-symbol interference (ISI). Then, each OFDM subcarrier $n$, belonging to a link between BS $k$ and user $u$, is subject to flat fading, path loss and shadowing with channel gains $\boldsymbol{G}=\left\{G_{u, n, k}\right\}$. In addition, the signals suffer from AWGN noise, which is Gaussian distributed with zero-mean and variance $\sigma^{2}$ and the co-channel interference power $\boldsymbol{I}=\left\{I_{u, n, k}\right\}$, which is defined as

$$
I_{u, n, k}=\sum_{i \neq k} G_{u, n, i} P_{v, n, i}, u=1, . ., U ; v \neq u,
$$

where: $G_{u, n, i}$ is the channel gain between the $i$ th interfering BS and user $u$ in cell $k$ and $P_{v, n, i}$ is the transmit power of BS $i$ on subcarrier $n$ assigned to user $v \neq u$, which is allocated to BS $i . \boldsymbol{G}=\left\{G_{u, n, k}\right\}, \boldsymbol{I}=\left\{I_{u, n, k}\right\}$ and $\sigma^{2}$ are assumed to be known by the system. The allocation of subcarrier $n$ to user $u$ at BS $k$ is expressed by the 3-dimensional allocation array $\boldsymbol{C}=\left\{C_{u, n, k}\right\}$, where $C_{u, n, k}=\{1,0\}$ means that subcarrier $n$ is allocated $\{1\}$ or is not allocated $\{0\}$ to user $u$ served by BS $k$. Additionally, we use a user-to-cell allocation matrix $\boldsymbol{A}=\left\{A_{u, k}\right\}$, indicating that user $u$ is allocated $\{1\}$ or is not allocated $\{0\}$ to cell $k$. Bit allocation is indicated by $\boldsymbol{b}=\left\{b_{u, n, k}\right\}$, where $b_{u, n, k}$ expresses the number of bits per symbol on subcarrier $n$ allocated to user $u$ served by BS $k$. Transmit power allocation is indicated by $\boldsymbol{P}=\left\{P_{u, n, k}\right\}$. Modulation levels are restricted, for practical reasons, to three $M$-QAM schemes with $M=4,16$, and 64 so the number of bits per symbol $b=\log _{2} M$ is limited to $\{2,4,6\}$, i.e. $b_{\min }=2$ and $b_{\max }=6$. The user mobility is low (WLAN-like scenario) so that the Doppler spread can be neglected. This, together with the assumption of perfect time and frequency synchronization, gives a system free from inter-channel interference (ICI). Users are assumed to be uniformly distributed over the service area. Both BSs and user terminals are equipped with omni-directional antennas. 


\subsection{Power Control}

The key relation in the cellular OFDMA system, bounding the transmit powers of the co-channel subcarriers, and expressing the signal to noise and interference ratio (SNIR) at subcarrier $n$ allocated to user $u$ served by cell $k$, is the following

$$
S N I R_{u, n, k}=\frac{G_{u, n, k} P_{u, n, k}}{I_{u, n, k}+\sigma^{2}} .
$$

In order to provide the data rate of $b_{u, n, k}=\log _{2}\left(M_{u, n, k}\right)$ bits and error probability of $P e_{u}, S N I R_{u, n, k} \geq \gamma_{u, n, k}$ should hold, where the threshold $\gamma_{u, n, k}$ is defined as, 13

$$
\gamma_{u, n, k}=\Gamma_{u, n, k}\left(2^{b_{u, n, k}}-1\right) .
$$

The SNR gap $\Gamma_{u, n, k}$ for an uncoded system is defined using the well known Q-function as, 13.

$$
\Gamma_{u, n, k}=\frac{1}{3}\left[Q^{-1}\left(\frac{P e_{u}}{4}\right)\right]^{2}, n=1, . ., N ; k=1, . ., K .
$$

Rearranging $S N I R_{u, n, k} \geq \gamma_{u, n, k}$ using (1)-3) leads to a set of linear inequalities, which should be fulfilled for all subcarriers in all the cells in order to guarantee the required level of $S N I R: \gamma_{u, n, k}$. This set of linear inequalities can be expressed for each subcarrier $n$ in the matrix form as

$$
\mathbf{H}(n) \mathbf{p}(n) \geq \sigma^{2} \gamma(n), n=1, . ., N,
$$

where

$$
\mathbf{H}(n)=\left[\begin{array}{cccc}
G_{1, n, 1} & -\gamma_{1, n, 2} G_{1, n, 2} & \ldots & -\gamma_{1, n, \kappa} G_{1, n, \kappa} \\
-\gamma_{2, n, 1} G_{2, n, 1} & G_{2, n, 2} & \ddots & \vdots \\
\vdots & \ddots & \ddots & \vdots \\
-\gamma_{\kappa, n, 1} G_{\kappa, n, 1} & \ldots & \ldots & G_{\kappa, n, \kappa}
\end{array}\right]
$$

and

$$
\gamma(n)=\left[\gamma_{1, n, 1} \gamma_{2, n, 2} \ldots \gamma_{\kappa, n, \kappa}\right]^{T},
$$

where $\kappa$ is the number of cells using subcarrier $n$. The columns in (6) correspond to co-channel cells (at subcarrier $n$ ), while rows correspond to users allocated to these cells (one user per cell). Given $\mathbf{H}(n), \gamma(n), \sigma^{2}$ the goal is to find an allpositive BS transmit power vector $\mathbf{p}(n)=\left[p_{1}(n), p_{2}(n), \ldots, p_{\kappa}(n)\right]$ containing the transmit powers of each BS using the $n$th subcarrier. If such a solution exists it is called a feasible solution. As explained in [14], 15] in order to find a feasible solution we need to solve equation

$$
\mathbf{H}(n) \mathbf{p}(n)=\sigma^{2} \gamma(n), n=1, . ., N .
$$


If the solution $\mathbf{p}(n)$ is all-positive then it also satisfies (5) . Otherwise, no feasible solution exists, which means that we can not reuse subcarrier $n$ in $\kappa$ cells. Solving equation (8) can be based on the well-known Gaussian elimination process, [15]. Having $\mathbf{p}(n)$ and knowing allocations of users to cells $\boldsymbol{A}$, we can easily compose $\boldsymbol{P}=\left\{P_{u, n, k}\right\}$.

\subsection{Resource Allocation Problem}

The goal of the resource allocation algorithm is to find such allocation arrays $\boldsymbol{C}=$ $\left\{C_{u, n, k}\right\}, \boldsymbol{b}=\left\{b_{u, n, k}\right\}$ and $\boldsymbol{P}_{\min }=\min (\boldsymbol{P})$ so that the user's traffic requirements are met at the cost of minimum total transmit power. The Allocation Problem can be formulated as follows

$$
\min \sum_{u=1}^{U} \sum_{n=1}^{N} \sum_{k=1}^{K} C_{u, n, k} P_{u, n, k}
$$

subject to

$$
\begin{gathered}
R_{u} \leq o R_{u}=\sum_{n=1}^{N} \sum_{k=1}^{K} C_{u, n, k} b_{u, n, k} ; u=1, . ., U, \\
P^{\max } \geq o P_{k}=\sum_{u=1}^{U} \sum_{n=1}^{N} C_{u, n, k} P_{u, n, k} ; k=1, . ., K, \\
\sum_{u=1}^{U} C_{u, n, k} \leq 1 ; n=1, . ., N ; k=1, . ., K, \\
C_{u, n, k} \in\{0,1\}, u=1, . ., U ; n=1, . ., N ; k=1, . ., K .
\end{gathered}
$$

Constraint (10) expresses user data rate requirement $R_{u}$ vs. offered data rate $o R_{u}$, constraint (11) is a limitation of a resulting transmit power $o P_{k}$, constraint (12) indicates that a subcarrier can be allocated to at most one user within a cell. Problem (9)-(13) is a 3-dimensional allocation problem, in which entries in the cost array $\boldsymbol{P}=\left\{P_{u, n, k}\right\}$ are mutually dependent due to CCI, as can be seen in relations (1)-(2), which is a major difficulty. This is also a non-linear, combinatorial optimization problem, since bits may take only integer values and the cost function is non-linear in one of the variables of interest ( $\boldsymbol{P}$ depends non-linearly on the number of bits $\boldsymbol{b}$ ). Therefore, in order to solve the problem (9)-(13), we resort to a suboptimal heuristic algorithm. 


\section{Proposed Solution}

The optimization problem stated in the previous section aims to minimize the sum transmit power of the entire system. Such general objective is fair regarding system-wide resource distribution, but the algorithm solving it closely would be difficult to implement in a cellular system. This is because a cellular system spanning usually over a service area of several (often more) cells has its dynamics caused by changes of the traffic and channel conditions due to new users arrival, users departure and users mobility in various places within a service area. These dynamics of a cellular wireless system are clearly more visible than that of fixed xDSL-like systems described in [9], [10]. Therefore, we take the following directions in designing the allocation algorithm:

- The (re)allocation of certain resources (not for entire-system) is triggered by the change of channel/traffic conditions of a single user.

- Not only the involved user has its resources allocated but also all the other users belonging to the candidate cell. This is done to exploit locally (within the candidate cell) multiuser diversity.

- Resources in all the other cells are not reallocated except the transmit power, which must be tuned to protect quality of the already existing and newly accessing links. Moreover, checking the existence of such power setting that satisfies all co-channel users, is a key part of admission control mechanism. A new user is admitted to the system only if its QoS requirement is fulfilled and the QoS of all the already existing connections is maintained at the acceptable cost.

In the following, we describe the suboptimal algorithm, which overcomes the main difficulty of the original allocation problem, namely the mutual dependency (due to CCI) of the entries in the cost matrix and which follows the abovepresented design directions. The solution consists of three steps, where in each step we allocate different resources. We start to determine the list of preferred cells $k_{\text {pref }}$ (sorted in descending order from the best one to the worst) for a considered user $\bar{u}$. For the first and not yet verified (for admission) cell $l$ on the list, we check the necessary condition for admission that is if the sum of minimal number of subcarriers already allocated to cell $l$ and required by a considered user to satisfy its rate requirement does not exceed the number of subcarriers available in the system, $N$. If it is not fulfilled then another cell on the $k_{\text {pref }}$ list is tried (adaptive cell selection). If the necessary condition is fulfilled then, for cell $l$, we allocate subcarriers to users, including the new user and already existing users (within cell $l$ ). This Step 2 is clearly more sensitive to local per-subcarrier channel gain levels. Finally, in Step 3 we set the modulation level (bit loading) and the power level for each subcarrier allocated to a user. This last part requires verification of existence of a feasible power vector, (5)-(8). After Step 3, we check the necessary condition for admission i.e. whether the QoS objectives of all the users are met. If so, the new user is allocated to the system and new resource allocations are applied. Otherwise the next cell on the list $k_{\text {pref }}$ is tried until we 
check all $k_{\text {checked }}^{\max }$ cells. If the necessary and sufficient conditions are not met for any of the best $k_{\text {checked }}^{\text {max }}$ cells, user $\bar{u}$ is blocked.

The "master" algorithm for resource allocation and admission control of a new user is depicted below.

Resource allocations for existing users : $\boldsymbol{A}, \boldsymbol{C}, \boldsymbol{b}, \boldsymbol{P}_{\min }$

Temporal resource allocations for new and existing users:

$\boldsymbol{A}_{\text {temp }}=\boldsymbol{A}, \boldsymbol{C}_{\text {temp }}=\boldsymbol{C}, \boldsymbol{b}_{\text {temp }}=\boldsymbol{b}, \boldsymbol{P}_{\text {min }}^{\text {temp }}=\boldsymbol{P}_{\text {min }}$

$s_{u}^{\min }=\left\lceil R_{u} / b_{\max }\right\rceil$ is a min number of subcarriers required to satisfy $R_{u}$

$s_{k}^{\text {min }}=\sum_{u \in \text { cellk }} s_{u}^{\text {min }}$ is a min number of subcarriers allocated already to cell $k$

For each new user $\bar{u}$ with : $R_{\bar{u}}, P e_{\bar{u}}, G_{\bar{u}, n, k}, I_{\bar{u}, n, k}$

Satisfaction indicator: satisf $=0$

Number of tried cells: $k_{\text {checked }}=0$

Step 1: find a list of preferred cells $k_{\text {pref }}$ for user $\bar{u}$

Cell index: $i=1$

While satisf $=0$ and $k_{\text {checked }}<k_{\text {checked }}^{\text {max }}$

$l=k_{\text {pref }}(i)$

// verification of the necessary condition for admission

If $\left(s_{\bar{u}}^{\text {min }}+s_{l}^{\text {min }} \leq N\right)$

update $\boldsymbol{A}_{t e m p}: A_{\bar{u}, l}^{t e m p}=1$

Step 2: find allocation of subcarriers to users in cell $l$, update $\boldsymbol{C}_{\text {temp }}$

Step 3: per involved subcarrier find allocation of: bits in cell $l$

and power levels in all cells; update $\boldsymbol{b}_{t e m p}, \boldsymbol{P}_{m i n}^{\text {temp }}$

// verification of the sufficient condition for admission

If $o R_{u} \geq R_{u}$ and $o P e_{u} \leq P e_{u}$ and $o P_{k} \leq P_{\max }, u=1, \ldots, U$ satisf $=1$

Else

satisf $=0 ; k_{\text {checked }}=k_{\text {checked }}+1 ; i=i+1$

End

Else

satisf $=0 ; k_{\text {checked }}=k_{\text {checked }}+1 ; i=i+1$

End

// final user admission or blocking

If satisf $=1$

Allow user $\bar{u}$ to the system:

$$
\boldsymbol{A}=\boldsymbol{A}_{t e m p}, \boldsymbol{C}=\boldsymbol{C}_{t e m p}, \boldsymbol{b}=\boldsymbol{b}_{t e m p}, \boldsymbol{P}_{\text {min }}=\boldsymbol{P}_{\min }^{t e m p}
$$

Else count user $\bar{u}$ as blocked

End

End

End

In the following, the algorithm's steps are discussed in more details.

\subsection{Step 1: User to Cell (Base Station) Allocation}

The basic idea behind "master" algorithm's Step 1 is to produce a list of preferred cells $k_{\text {pref }}$ based on the criterion of best average (over all subcarriers) normalized channel gain and interference level, which is particularly important in cellular environments and is usually not considered in classical cell selection schemes (e.g. based on received signal level or distance). We allow a user to be 
served by one base station only, in order to avoid prospective problems due to synchronization misalignments expected to arise if a user was connected to multiple base stations using different subcarriers. The average (over all subcarriers) normalized channel gain for a user $u$ at cell $k$ can be expressed by

$$
\bar{T}_{u, k}=\frac{1}{N} \sum_{n=1}^{N} T_{u, n, k},
$$

where the normalized channel gain is defined as

$$
T_{u, n, k}=\frac{G_{u, n, k}}{\left(I_{u, n, k}+\sigma^{2}\right) \Gamma_{u, n, k}} .
$$

The list of preferred cells $k_{\text {pref }}$ for user $u$ is a vector of cell indices to the sorted (in descending order) normalized channel gains as shown below

$$
k_{\text {pref }}=\arg \operatorname{sort}_{k} \bar{T}_{u, k}
$$

\subsection{Step 2: Subcarrier to User Allocation}

Having temporarily allocated user $\bar{u}$ to a cell $l$ the task is to allocate subcarriers for this user. In order to exploit multiuser diversity, the allocation of subcarriers for user $\bar{u}$ is done together with reallocating subcarriers of users already present in cell $l$. For this purpose we apply a slightly modified version of a two-phase algorithm proposed in [4].

In phase $A$, we determine the number of subcarriers each user would get (proportionally to its rate requirement) by verifying the relative reduction of cell transmit power after allocation of additional subcarrier. The modification of the original algorithm includes using $s_{u} \leq s_{u}^{\max }$ as a stop-criterion in increasing the number of subcarriers for a user, since allocating more subcarriers than a user may operate leads to blocking these subcarriers for other users where they might be better exploited.

//Phase A: determine the number of subcarriers for each $u$ $s_{u}^{\max }=\left\lceil R_{u} / b_{\min }\right\rceil ; s_{u}=s_{u}^{\min }=\left\lceil R_{u} / b_{\max }\right\rceil$ for each $u$

While $\sum_{u=1}^{U} s_{u}<N$ and $s_{u} \leq s_{u}^{\max }$ do

For $u \in$ cell $l$

$$
\begin{aligned}
& \bar{P}_{u}=s_{u}\left(2^{R_{u} / s_{u}}-1\right) / \bar{T}_{u, l} \text { is the average power a user } \\
& \quad \text { would require to transmit using } s_{u} \text { subcarriers in cell } l \\
& \bar{P}_{u}^{\text {new }}=\left(s_{u}+1\right)\left(2^{R_{u} /\left(s_{u}+1\right)}-1\right) \bar{T}_{u, l} \\
& \Delta P_{u}=\bar{P}_{u}-\bar{P}_{u}^{n e w} \\
& w=\arg \max _{u} \Delta P_{u} \\
& s_{w}=s_{w}+1 \\
& \bar{P}_{w}=\bar{P}_{w}+1 \\
& \text { End }
\end{aligned}
$$$$
\text { would require to transmit using } s_{u} \text { subcarriers in cell } l
$$

End 
The output of this phase is the number of subcarriers $\left\{s_{u}\right\}$ that each user in cell $l$ should use. Knowing this, in phase $B$ we allocate particular subcarriers for particular users within a given cell. The algorithm is based on a simple greedy routine distributing subcarriers among users based on best normalized channel gains. Such approach is motivated by a simple observation that users with good channels require less transmit power, thus causes less CCI and therefore have higher chances for admission. Various options of this routine have been described in many papers, including [5], 4] and [3]. An alternative strategy is to use the optimal Hungarian algorithm or to improve the greedy assignment by swapping subcarriers between users as in [5], [3]. These methods, however, are not considered here due to their increased computational complexity.

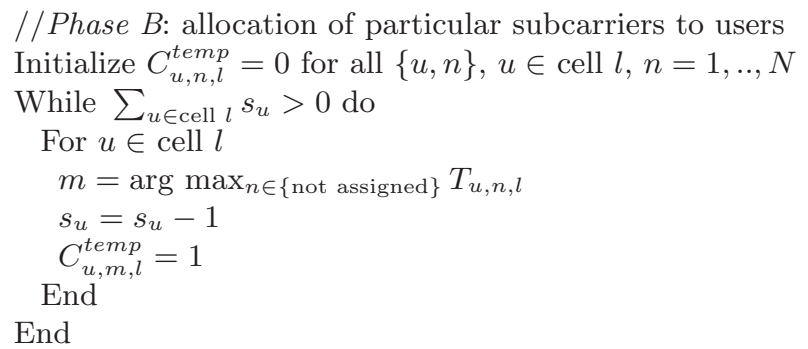

\subsection{Step 3: Bit and Power Allocation per Subcarrier}

After having temporarily allocated users to cells according to $\boldsymbol{A}_{\text {temp }}$ and subcarriers to users according to $\boldsymbol{C}_{\text {temp }}$, the bit and power allocation boils down to single-user bit loading in the presence of background CCI. This algorithm step is similar to the one proposed in [9], [10] with the exception that it does not run over all subcarriers (and in case of a cellular system also over all base stations) since these allocations have been done already in previous steps. In effect, the computational load is reduced. Therefore, for each user $u$ allocated in cell $l$, within a set of subcarriers allocated to user $u$, the algorithm increases the modulation level by one step (which corresponds to increasing the number of bits from $b_{u, n, l}$ to $b_{u, n, l}^{n e w}$ ) on a subcarrier, where it requires least transmit power increase. Subsequently, transmit power is set in a given cell and adjusted in all co-channel cells in order to maintain QoS of already existing connections. The procedure is repeated until user's rate requirement is fulfilled or the system is saturated for this user, which means that all subcarriers are not feasible (i.e. either maximal modulation level is already achieved or there is no feasible power setting over all co-channel cells). The aim of this routine is to determine the temporary number of bits for users within considered cell $l$ and to set the temporary power levels within the cell $l$ as well as within all the co-channel cells reusing the same subcarriers as cell $l$. Both bit and power allocations are parts of the all-system allocation arrays $\boldsymbol{b}_{\text {temp }}$ and $\boldsymbol{P}_{\text {min }}^{\text {temp }}$, in which only the involved entries are modified. The routine is outlined below. 


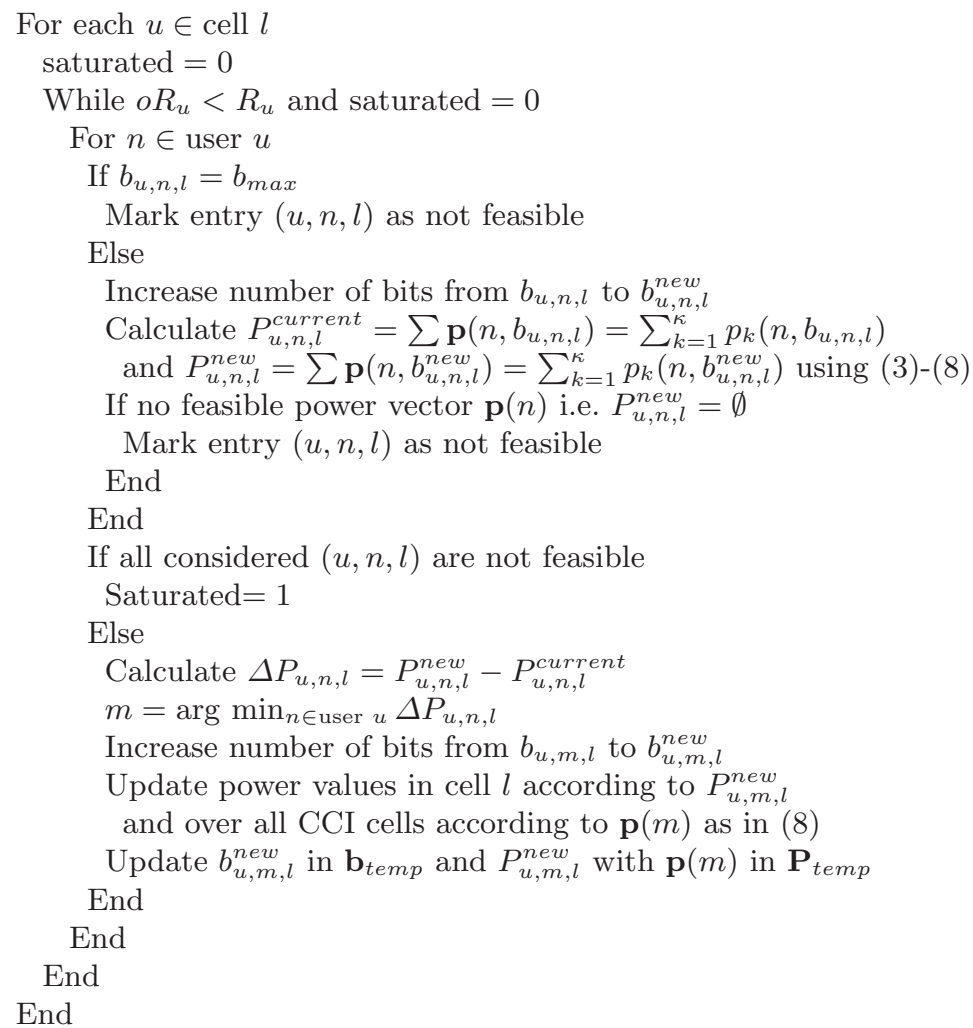

\section{Numerical Experiments}

The key point of this paper is the performance evaluation of the proposed algorithms, which is done by numerical experiments. We simulated the downlink of a cellular network consisting of $K=19$ cells, each with one BS having one omni-directional antenna, as depicted in Figure 2 with users appearing one-byone at random locations within the system service area. The following system parameters were fixed: system bandwidth $B W=5 \mathrm{MHz}$, mean Ricean K-factor: $4.9 \mathrm{~dB}$, and variance of the Gaussian distributed power variations due to shadowing: $6 \mathrm{~dB}$. Each user is assumed to require the same data rate $R_{u}=R$, and error probability $P e_{u}=P e$, though the model allows to set $R_{u}$ and $P e_{u}$ per user individually. We have evaluated the influence of changing the following system parameters: a) mean rms delay spread $r d s=\{50,100,200,500,1000,1500\} \mathrm{ns}$, b) data rate required by a single user $R=\{0.5,1,2,4,8\} \mathrm{Mbit} / \mathrm{s}$ and c) path loss exponent $\alpha=\{1.5,2,2.5,3,3.5,4\}$.

The following schemes have been taken for comparison:

- proposed: three modulation levels are applied, namely 4-QAM, 16-QAM and 64-QAM, adaptive cell selection (ACS) is allowed with $k_{\text {checked }}^{\max }=5$, 


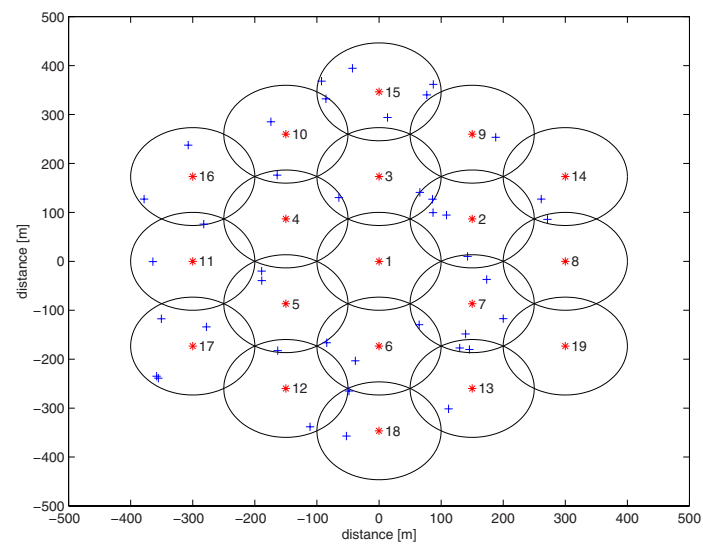

Fig. 2. Cellular network used in the simulations. Example distribution of $U=40$ users (indicated by crosses) over $K=19$ cells (base stations indicated by stars)

- proposed no ACS: three modulation levels are applied, namely 4-QAM, 16QAM and 64-QAM, ACS is not allowed i.e. $k_{\text {checked }}^{\text {max }}=1$,

- proposed fixed no ACS: modulation level is fixed to 4-QAM, ACS is not allowed i.e. $k_{\text {checked }}^{\max }=1$,

- FDMA: users are allocated to base stations as in proposed fixed scheme, subcarriers are allocated to users in a fixed classical FDMA way, according only to their data rate requirements; modulation level is 4-QAM. Power control is used as in proposed scheme in order to provide and maintain required QoS. ACS is not allowed i.e. $k_{\text {checked }}^{\text {max }}=1$.

We have used the following metrics for performance comparison: (a) total data rate offered, which is the sum offered data rate over all users $\sum_{u} o R_{u}$ provided that the QoS requirement is met for all users (both new and existing) and (b) blocking probability, which is a probability that a new user will be blocked due to insufficient resources to support required QoS. The performance comparison metrics, averaged over 100 network realizations, are gathered at the reference load of $50 \mathrm{Mbit} / \mathrm{s}$, which indicate the total data rate required in the network. In the simulations performed, no wrap-around technique was applied in order to reflect a small hot-spot network (WLAN-like), where the coverage is usually limited to a couple of base stations.

\subsection{Influence of Delay Spread}

The comparison of the considered schemes for various mean rms delay spread $r d s=\{50,100,200,500,1000,1500\}$ ns is depicted in Figure 3 It can be observed that the total data rate offered of the proposed, proposed no ACS and proposed fixed no $A C S$ slightly degrades with the increased $r d s$ but are above the required reference data rate of $50 \mathrm{Mbit} / \mathrm{s}$. This can be explained by the fact that the 

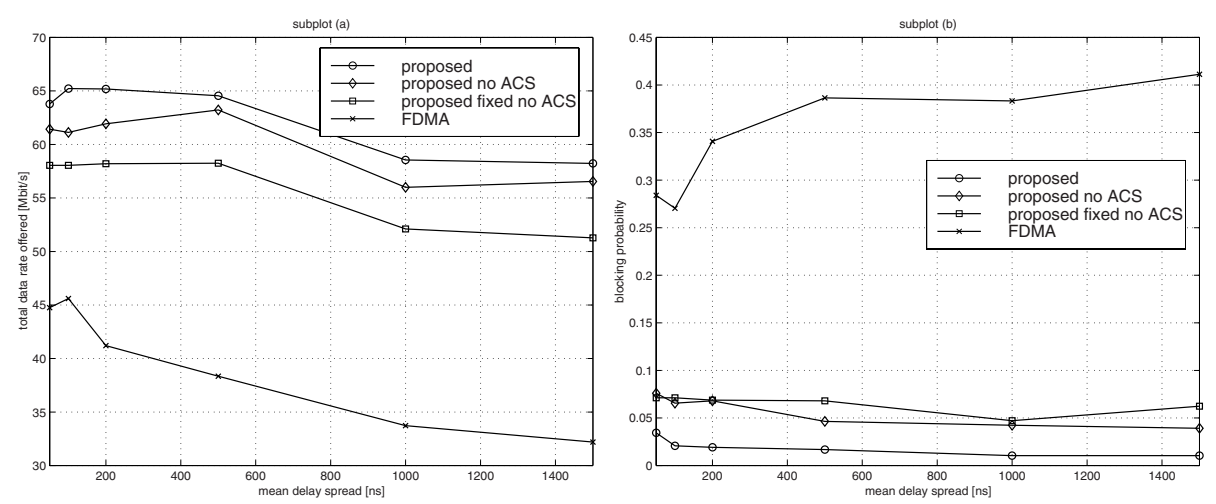

Fig. 3. Influence of various mean delay spread $\mathrm{rms}$. The other system parameters are: $N=16$ for $r d s=\{50,100,200,500\}$ ns and $N=32$ for $r d s=\{1000,1500\} \mathrm{ns}, \alpha=4$, $R=2 \mathrm{Mbit} / \mathrm{s}$

offered data rates have a granularity determined by the number of subcarriers and the three modulation levels. For $r d s=\{50,100,200,500\}, N=16$ thus granularity is lower than for $r d s=\{1000,1500\}$, where $N=32$. For $N=16$ users get higher data rates than the required $2 \mathrm{Mbit} / \mathrm{s}$ (per single user) because it is not possible to compose exactly $2 \mathrm{Mbit} / \mathrm{s}$. For $N=32$ we are closer to the required $2 \mathrm{Mbit} / \mathrm{s}$. What is maybe more interesting is that with the increased $r d s$ (i.e. increased frequency selectivity), the blocking probability of the proposed and proposed no ACS actually improves, while in case of FDMA it degrades. This can be explained by the fact that the more frequency selectivity we have, the more we can gain from multiuser diversity. For fixed schemes (FDMA), on the other hand, the multiuser diversity is not exploited and thus a higher $r d s$ has a negative effect. It is interesting to note that blocking probability for the proposed fixed no ACS is relatively constant. This would mean that in this case blocking probability is improved rather by the application of adaptive modulation (as in proposed and proposed no ACS) than by adaptive subcarrier allocation applied to all users in the considered cell.

\subsection{Influence of Data Rate Required by a Single User}

The performance comparison of the considered schemes for various data rates $R$ required by a single user, where $R=\{0.5,1,2,4,8\}$ [Mbit/s] is depicted in Figure 4. The observations in this case are similar to the previous observations on frequency selectivity. Also here, the total data rate offered of the proposed, proposed no ACS slightly degrades with the increased $R$ but is above the required reference data rate of $50 \mathrm{Mbit} / \mathrm{s}$. For proposed fixed no ACS, offered data rate drops quite rapidly, which indicates the importance of adaptive modulation (such drop is not observed in case of schemes employing adaptive modulation). When looking at the blocking probability, in case of proposed fixed no ACS, it 

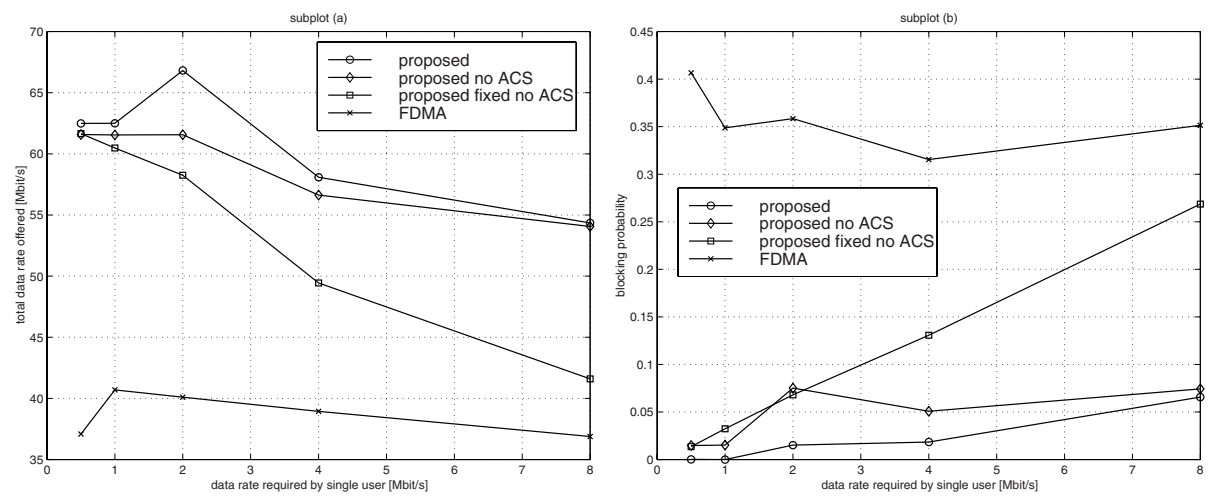

Fig. 4. Influence of various data rates required by a single user $R$. The other system parameters are: $N=16, r d s=200 \mathrm{~ns}, \alpha=4$

also degrades rapidly with the increased data rate per user. For proposed and proposed no ACS degradation is also observed but it is moderate. This can be explained by the fact that for proposed fixed no ACS, the better performance for low data rate per single user is due to multiuser diversity exploited by adaptive subcarrier allocation (and reallocation of users served by a considered cell), since this method can not exploit channel variability with the use of adaptive modulation. At a constant reference load of $50 \mathrm{Mbit} / \mathrm{s}$, lower data rate requirement per single user means more users per cell. This obviously increases the gain from multiuser diversity, since we have more options (corresponding to users) to find good subcarriers. Heavy users (such as $8 \mathrm{Mbit} / \mathrm{s}$ in this case) result in low number of users per cell and thus lower multiuser diversity.

\subsection{Influence of Path Loss}

The performance comparison of the considered schemes for various path loss conditions $\alpha=\{1.5,2,2.5,3,3.5,4\}$ is depicted in Figure 5. It can be observed that path loss exponent influences all the considered schemes in the same way: low $\alpha$ means poor shielding from CCI and thus low offered data rates and high blocking probability, while high $\alpha$ means good shieling from CCI thus increased offered data rates and decreased blocking probabilities.

\section{$5 \quad$ Summary}

In this paper we addressed a 3-dimensional problem of allocating users, base stations, subcarriers, bits and transmit power in a cellular multi-user OFDMA system. We have proposed a modification of the algorithms described in [12] to solve this allocation problem. The modification includes possibility to perform adaptive cell selection in order to find suitable cell to serve a user. In addition, we 

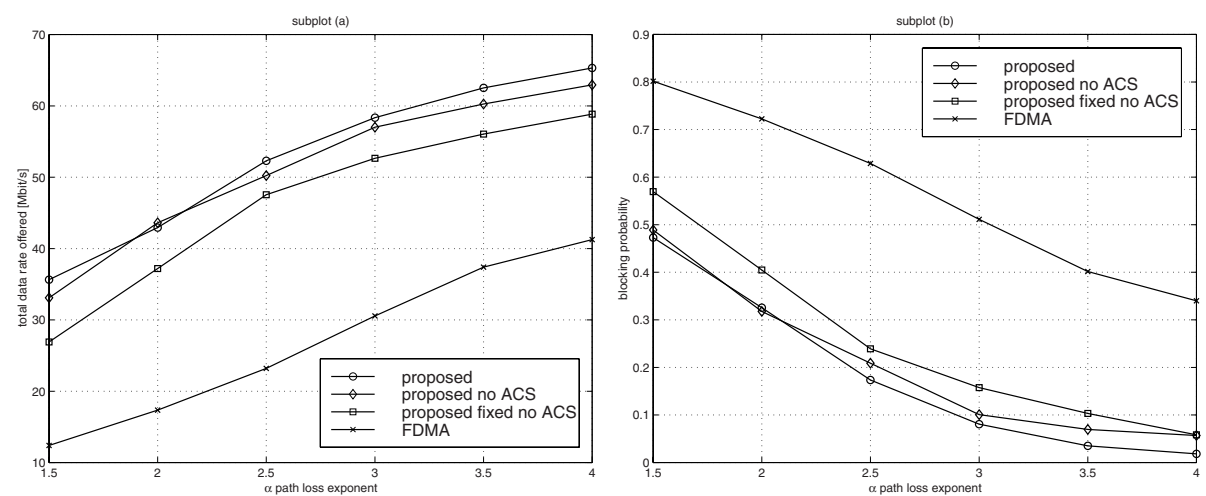

Fig. 5. Influence of various path loss exponent $\alpha$. The other system parameters are: $N=16, r d s=200 \mathrm{~ns}, R=2 \mathrm{Mbit} / \mathrm{s}$

have evaluated the performance of the various options of the proposed algorithm in different scenario settings.

In all the considered cases, the best performance (offered data rate and blocking probability) is achieved with the proposed algorithm, which utilizes both adaptive cell selection and adaptive modulation. The worst performance has been observed with FDMA scheme. We have observed that the proposed algorithm improves its performance with increased frequency selectivity, which is disastrous for fixed FDMA. Moreover, we have observed that the more users per cell we have, the more we can exploit multiuser diversity with our adaptive techniques. This indicates the trade-off between allowed range of traffic profiles and offered system capacity. Another indication regarding the allowed traffic profiles is that it is important to match the required data rates to granularity offered by a systems in order to offer just-as-required data rates.

Potential implementations of the proposed schemes include mainly lowmobility, frequency selective, cellular radio access systems, such as OFDM-based WLAN (e.g. 802.11a, HiperLAN/2) or Broadband Wireless Access (e.g. 802.16).

\section{References}

1. J. Zander, "Radio Resource Management in Future Wireless Networks: Requirements and Limitations", IEEE Communications Magazie, August 1997.

2. G.J. Pottie, "System Design Choices in Personal Communications", IEEE Personal Communications, October 1995.

3. C.Y. Wong et al, "A Real-time Sub-carrier Allocation Scheme for Multiple Access Downlink OFDM Transmission", IEEE VTC'99.

4. D. Kivanc, H. Liu, "Subcarrier Allocation and Power Control for OFDMA", 34th Asilomar Conference on Signals, Systems and Computers, November 2000.

5. S. Pietrzyk, G.J.M. Janssen, "Multiuser Subcarrier Allocation for QoS Provision in the OFDMA Systems", IEEE VTC'02 Fall, September 2002, Vancouver, Canada. 
6. J.C.I. Chuang and N.R. Sollenberger, "Spectrum Resource Allocation for Wireless Packet Access with Application to Advance Cellular Internet Service", IEEE JSAC, August 1998.

7. Y. Zhang and K.B. Letaief, "Multiuser Subcarrier and Bit Allocation along with Adaptive Cell Selection for OFDM Transmission", IEEE ICC, 2002.

8. J. Li, H. Kim, Y. Lee and Y. Kim, "A Novel Broadband Wireless OFDMA Scheme for Downlink in Cellular Communications", IEEE WCNC, 2003.

9. J. Lee, R.V. Sonalkar, J.M. Cioffi, "Multi-User Discrete Bit-Loading for DMTbased DSL Systems", IEEE Globecom 2002.

10. G. Kulkarni, M. Srivastava, "Subcarrier and Bit Allocation Strategies for OFDMA based Wireless Ad Hoc Networks", IEEE Globecom 2002.

11. S. Pietrzyk, G.J.M. Janssen, "Subcarrier Allocation and Power Control for QoS Provision in the Presence of CCI for the Downlink of Cellular OFDMA Systems", IEEE VTC'03 Spring, April 2003, Jeju, Korea.

12. S. Pietrzyk, G.J.M. Janssen, "Radio Resource Allocation for Cellular Networks Based on OFDMA with QoS Guarantees", (paper accepted), IEEE Globecom, December 2004.

13. J.M. Cioffi, "A Multicarrier Primer", Nov. 1991.

14. N. Bambos, G.J. Pottie, "Power Control Based Admission Policies in Cellular Radio Networks", IEEE Globecom 1992.

15. S.C. Chen, N. Bambos, G.J. Pottie, "Admission Control Schemes for Wireless Communication Networks with Adjustable Transmitter Powers", IEEE Infocom 1994, Toronto. 